\title{
COMPARISON OF SEMI-AUTOMATIC AND AUTOMATIC SLICK DETECTION ALGORITHMS FOR JIYEH POWER STATION OIL SPILL, LEBANON
}

\author{
B. Osmanoglu ${ }^{\text {a, }}{ }^{,}$, C. Ozkan ${ }^{\text {b }}$, F. Sunar ${ }^{\mathrm{c}}$ \\ ${ }^{a}$ USRA - NASA Goddard Space Flight Center, USA - batuhan.osmanoglu@ nasa.gov \\ ${ }^{\mathrm{b}}$ Erciyes University, Kayseri, Turkey - cozkan@erciyes.edu.tr \\ ${ }^{c}$ Istanbul Technical University, Istanbul, Turkey - fsunar@itu.edu.tr
}

KEY WORDS: Oil spill, SAR

\begin{abstract}
:
After air strikes on July 14 and 15, 2006 the Jiyeh Power Station started leaking oil into the eastern Mediterranean Sea. The power station is located about $30 \mathrm{~km}$ south of Beirut and the slick covered about $170 \mathrm{~km}$ of coastline threatening the neighboring countries Turkey and Cyprus. Due to the ongoing conflict between Israel and Lebanon, cleaning efforts could not start immediately resulting in 12,000 to 15,000 tons of fuel oil leaking into the sea.

In this paper we compare results from automatic and semi-automatic slick detection algorithms. The automatic detection method combines the probabilities calculated for each pixel from each image to obtain a joint probability, minimizing the adverse effects of atmosphere on oil spill detection. The method can readily utilize X-, C- and L-band data where available. Furthermore wind and wave speed observations can be used for a more accurate analysis. For this study, we utilize Envisat ASAR ScanSAR data. A probability map is generated based on the radar backscatter, effect of wind and dampening value.

The semi-automatic algorithm is based on supervised classification. As a classifier, Artificial Neural Network Multilayer Perceptron (ANN MLP) classifier is used since it is more flexible and efficient than conventional maximum likelihood classifier for multisource and multi-temporal data. The learning algorithm for ANN MLP is chosen as the Levenberg-Marquardt (LM). Training and test data for supervised classification are composed from the textural information created from SAR images. This approach is semiautomatic because tuning the parameters of classifier and composing training data need a human interaction.

We point out the similarities and differences between the two methods and their results as well as underlining their advantages and disadvantages. Due to the lack of ground truth data, we compare obtained results to each other, as well as other published oil slick area assessments.
\end{abstract}

\section{INTRODUCTION}

Oil spills pose a threat to any nation that borders a body of water. Economical and environmental losses due to an oil spill are proportional to the volume of spill, and cause devastating consequences for the environment regardless of size. Timely detection and monitoring of spills are necessary to minimize these adverse effects.

Synthetic Aperture Radar (SAR) systems provide a viable option for oil slick monitoring. The intensity of SAR images depends on the surface roughness, which is altered in the event of an oil spill. Bragg scattering dominates the radar response over ocean surfaces (Bragg, 1913). Oil covering an ocean surface dampens surface waves, causing specular reflection of the radar wave. This ultimately results in reduced backscatter energy returning to the satellite. It is important, therefore, to have some wind to produce surface waves. Since SAR systems have a limited range of ideal wind speed and direction where they are most sensitive, they do not perform well for oil spill detection under very windy or very calm conditions.

Other methods for ocean monitoring and oil detection have been proposed over the years, including infrared remote sensing and hyperspectral imaging sensors (Brekke and Solberg, 2005). Each method presents different levels of uncertainty, and it is therefore possible to combine information from different data sources to reduce uncertainties. Results from different remote sensing sensors, as well as field observations, can be combined to provide a statistically better outcome than any individual resource.

In this paper we propose a fully automatic oil spill monitoring system that is capable of combining data from multiple SAR images. The focus of this paper is on radar imagery; the algorithm, however, can be expanded to optical imagery and ground measurements as discussed later. Moreover this technique is compared to a semi-automatic technique based on supervised classification of textural features. A brief background on the technical aspects is provided in Section 2. Results obtained from SAR data over the Jiyeh Power Station oil spill are discussed in Section 3.

\section{METHODS}

Synthetic Aperture Radar imagery is sensitive to surface roughness, which is altered in the event of an oil spill (Alpers and Huhnerfuss, 1988). Oil slicks change the smoothness of the ocean surface and appear darker compared to the surrounding oil-free ocean. The amount of dampening, however, is affected by wind and wave conditions. Furthermore, the speckle effect in SAR imagery limits the reliability of point measurements in the image, causing spurious results (Brekke and Solberg, 2005). In our approach we apply multi-step processing to limit the adverse effects of speckle, rather than filtering data with a speckle filter. 
There are many methods developed to detect oil spills from SAR intensity images: (1) machine learning and neural-network recognition (Kubat et al., 1998, Ozkan and Sunar, 2007, Ozkan et al., 2013), (2) frequency spectrum attenuation (Lombardini et al., 1989, Gade et al., 1998, Kim et al., 2010), (3) segmentation techniques (Barni et al., 1995, Solberg et al., 2007), and (4) slick feature extraction (Fiscella et al., 2000, Del Frate et al., 2000). Some algorithms also combine ocean drift models to assist data analysis (Espedal, 1999, Cheng et al., 2011). In addition to SAR intensity, co-polarization differences of multiple-polarization SAR data is also suggested for oil spill detection (Migliaccio et al., 2009). In this paper, we compare the results of an automatic and a semi-automatic oil detection method.

\subsection{Automatic Oil Detection}

The automatic method relies on combining the results of several different SAR analyses to generate a robust, automatic oil spill detector. It is also important to note that this approach can be seen as a framework for combining information from any geospatial observation (e.g. optical remote sensing, ground measurements). The proposed algorithm is composed of four calculation steps: (1) Dark object selection, (2) dampening factor calculation, (3) combination of probabilities, and (4) temporal analysis. Dark object selection is based on normalized radar cross section, where darker pixels get higher probabilities for oil contamination (Barni et al., 1995). Dampening factor is based on the current wind conditions and imaging parameters (Gade et al., 1998, Kim et al., 2010). Results from both analyses are then combined to provide a joint probability for the presence of an oil slick at each acquisition. The temporal probability is formed using multiple SAR acquisitions, resulting in a timevarying probability of oil slick over target area. All analyses in this paper are performed over intensity-calibrated, geocoded SAR imagery. Images are calibrated to normalized radar cross section (NRCS). The imagery is resampled to a common geometry using a sinc interpolator, and land-masked using Global, Self-consistent, Hierarchical, High-Resolution Shoreline data (GSHHS) (Wessel and Smith, 1996).

\subsubsection{Dark Object Selection}

The first step of the algorithm is a simple dark object selection routine based on intensity thresholding. At this step, dark areas of the image are assigned a higher probability for an oil spill.

In this step we apply an adaptive threshold to select about $10 \%$ of the imaged area. For the Envisat Scansar images, this corresponds to $2500 \mathrm{~km}^{2}$, which is much larger than any expected oil spill. A probability value for each pixel is assigned based on its intensity such that:

$$
\begin{aligned}
& P\left(\left.W\right|_{0}\right)=\left(\begin{array}{lll}
0 & \left.\min \left({ }_{0}\right)\right)
\end{array}\right) /\left(\begin{array}{l}
T \min \left({ }_{0}\right)
\end{array}\right) \\
& P\left(O \mid{ }_{0}\right)=1 \quad P\left(W \mid{ }_{0}\right)
\end{aligned}
$$

where $\mathrm{P}\left(\mathrm{W} \mid \sigma_{0}\right)$ is probability of oil-free water given the NRCS, $\mathrm{T}$ is the threshold, and $\mathrm{P}(\mathrm{O} \mid \sigma 0)$ is the probability of oil given the NRCS is the complement of $\mathrm{P}(\mathrm{W} \mid \sigma 0)$. The $\mathrm{P}(\mathrm{W} \mid \sigma 0)$ is modified by bringing all larger values to 1 , constraining the probability values between 0 and 1 . We then combine this map with the estimated wind speeds to remove dark areas due to low wind speeds. Because a low wind area cannot contain any useful information about oil slicks, the joint probability of these two different events are simply their multiplication:
$\mathscr{P}\left(\mathrm{O} \mid \sigma_{0}, u_{0}\right)=\mathscr{P}\left(\mathrm{O} \mid \sigma_{0}\right) \mathscr{P}\left(\mathrm{O} \mid u_{0}\right)$

where $\mathrm{u}_{0}$ indicates the estimated wind speed.

\subsubsection{Dampening Factor}

It is possible to calculate dampening factors due to presence of oil (Gade et al., 1998, Kim et al., 2010). The estimated dampening factor varies with wind speed and direction as a function of the Bragg wave numbers as shown in Figure 1. The Bragg wave number is defined as (Gade et al., 1998):

$k_{B}=2 k_{0} \sin$

Where $k_{B}$ is the Bragg wave number, $k_{0}$ is the radar wave number and $\theta$ is the incidence angle. Figure 1 shows the maximum expected dampening amount which would be observed when the angle between the wind, and radar wave is zero.

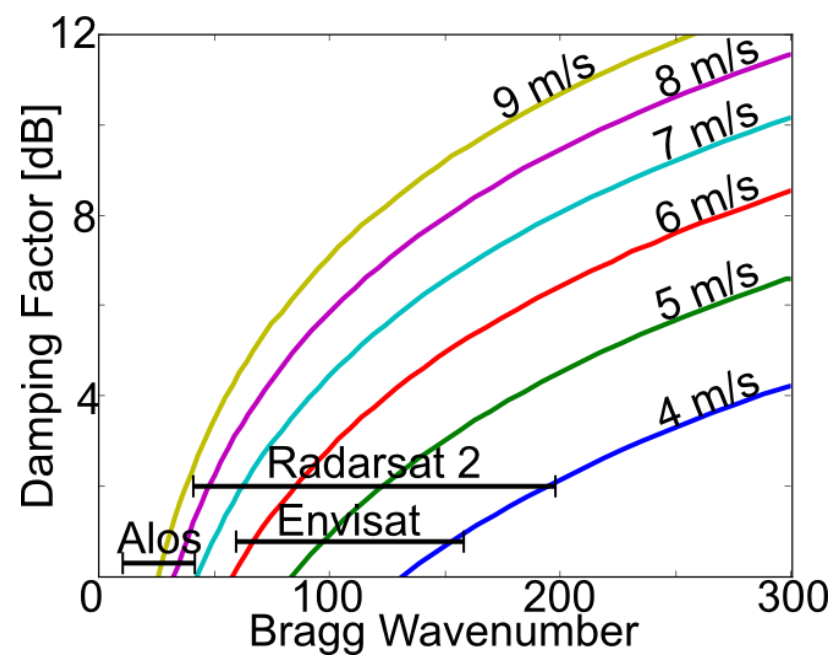

Figure 1. Estimated dampening factor as a function of Bragg wave number for common SAR sensors. The theoretical range of SAR systems used in the system are marked with horizontal lines. Each curve represents a different wind velocity.

Wind speed and the angle between the radar look direction and wind play an important role in the dampening factor. Figure 2 shows a plot of expected dampening factors at speeds between $2.5 \mathrm{~m} / \mathrm{s}$ and $12.5 \mathrm{~m} / \mathrm{s}$. The relative wind direction is plotted at counter-clockwise increasing angles, starting from zero at the horizontal axis. In this study we use the wind speed and direction data from QuickScat satellite. 


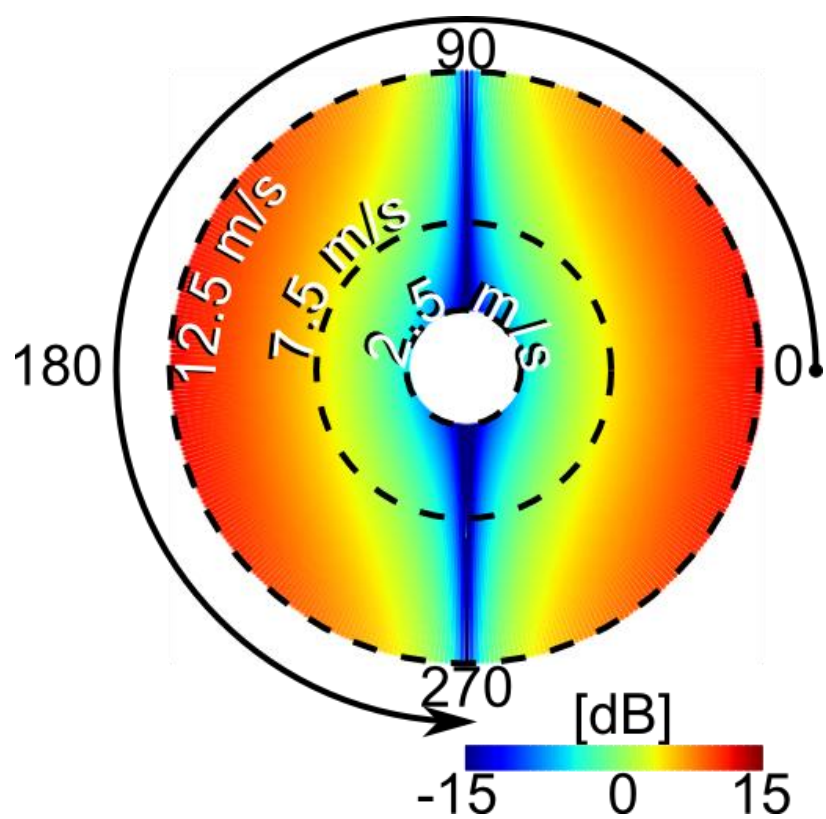

Figure 2. Estimated dampening factor plotted against the relative angle between radar and wind direction. Radial distance from the center indicates the wind speed.

\subsubsection{Joint Probability}

The oil spill probability based on the dark object $P(O \mid n)$ and dampening factor $\mathrm{P}(\mathrm{O} \mid \mathrm{d})$ can be combined following the Bayesian theory:

$$
J P(O)=\frac{P(O \mid n) P(O \mid d)}{P(O \mid n) P(O \mid d)+P(W \mid n) P(W \mid d)}
$$

where J P $(\mathrm{O})$ is the joint probability for oil, $\mathrm{P}(\mathrm{O} \mid \mathrm{n})$, is the probability of oil given the NRCS, $\mathrm{P}(\mathrm{O} \mid \mathrm{d})$ is the probability of oil given the dampening factor analysis, $\mathrm{P}(\mathrm{W} \mid \mathrm{n})$ is the probability of clear water given the NRCS, and $\mathrm{P}(\mathrm{W} \mid \mathrm{d})$ is the probability of clear water given the dampening factor analysis.

\subsubsection{Temporal Probability}

It is possible to remove false positives by constraining the results from individual images with a temporal relationship. The slick is expected to move around with the influence of surface waves. It is also expected to evaporate and sink as it weathers. For this analysis we define a simple $a$ priori relation, and expect the slick to stay in a $7.5 \mathrm{~km}$ neighborhood with a continuously reducing probability for a week. After one week, the temporal probability defaults to equal probabilities for oil slick and clean surface. Temporal probability is applied forward and backward in time.

\subsection{Semi-Automatic Oil Detection}

The semi-automatic scheme is based on the supervised classification, for which the selection of dark features is so important that the success of the approach mainly depends on it. Because of the speckle noise of SAR imaging, pixel based classification is not appropriate. Therefore, textural information, variation of the pixel intensities in a specific image area, may be used to take into account the spatial relationship. As the most well known texture measures, Haralick features are computed by gray level co-occurence matrix (GLCM) based on image intensities (Haralick et al., 1973). A co-occurrence matrix is like a one way contingency matrix which defines a two dimensional histogram of intensity values for a pair of image pixels which are separated by a fixed spatial relationship. Of the many texture measures, mean, variance, homogeneity and angular second moment are chosen to become features in classification (Assilzadeh and Mansor, 2001). GLCM is obtained from $5 \times 5$ image windows of SAR image filtered with median and lowpass filters. These grey level co-occurrence matrixes (GLCM) based textural features are computed from the oil and look-alike regions using $5 \times 5$ kernels. Consequently, the input data dimension is set as four.

Homogeneity $=\frac{P_{i j}}{i, j}$

Angular Second Moment $={ }_{i, j} P_{i j}^{2}$

Mean $=\underset{i, j}{(i, j) P_{i j}}$

Variance $=[i, j-\text { Mean }]^{2} P_{i j}$ $i, j$

where, $\mathrm{P}_{\mathrm{ij}}$; is the normalized GLCM value. So, input feature space is homogeneity, angular second moment, mean and variance, the output classes is oil and look-alike. Further details on this method are presented in an earlier study, utilizing the same data set (Ozkan et al., 2013).

\section{RESULTS AND DISCUSSION}

In order to test this hypothesis we analysed data from nine different Envisat ASAR images acquired between August 3rd and September 4th 2006, using a fully automatic algorithm. The algorithm relies on selecting dark objects in two different steps: (1) pixel-wise, and (2) spatially for each image. Furthermore, a temporal probability was calculated to combine results from different images.

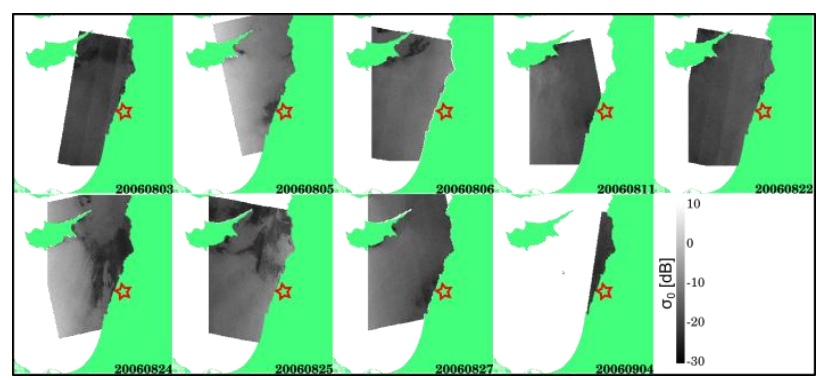

Figure 3. Envisat ASAR Wide Swath images acquired over the study area during the oil spill. Land areas are masked with light green color. The red star denotes the approximate location of the Jiyeh Power Plant.

The images in Figure 3 are calibrated to obtain normalized radar cross section using the ESA Basic Envisat SAR Toolbox. It is clear that even though the images are calibrated the same way, images have varying levels of contrast. The changes in contrast are likely related to the surface roughness conditions, which may be partly related to varying wind speeds.

Quickscat satellite provides wind direction and speed measurements over the oceans using a scatterometer. Quickscat covers about $90 \%$ of the globe every day, and visits the same 
area twice. Figure 4 represents the Quickscat measurements over the study area. In general wind speeds are about 10 knots and follow a SSW (South - South West) direction near the Lebanese coast.

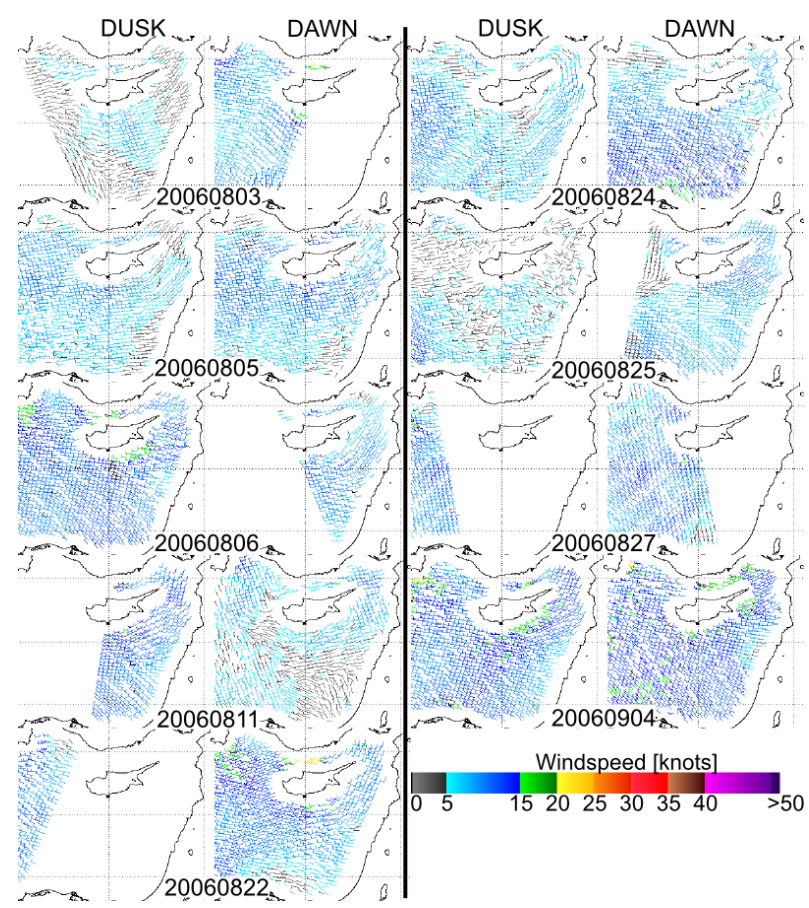

Figure 4. Quickscat wind measurements. The direction of the barbs indicate the wind direction while their color provide the windspeed information. The black barbs mark unreliable data due to rain. Quickscat visits the same area twice a day, at dusk and dawn.

Using the method described in Section 2, and the data presented in Figures 3 and 4, results were obtained for the Jiyeh oil spill. The results are presented in Figures 5 and 6.

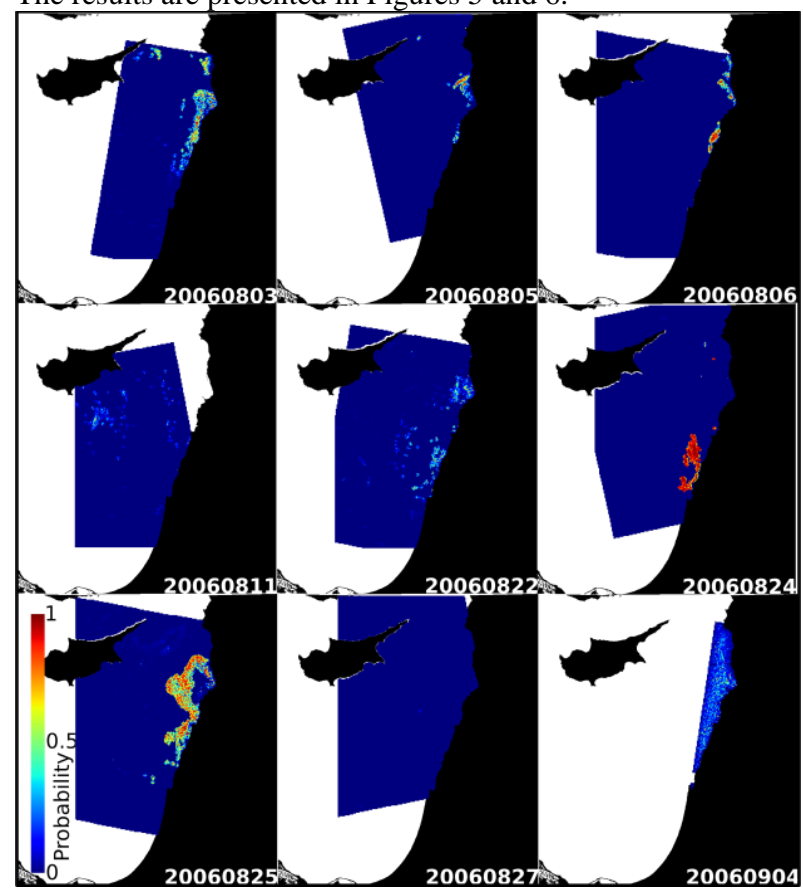

Figure 5. Results obtained from the automatic oil spill detection algorithm.

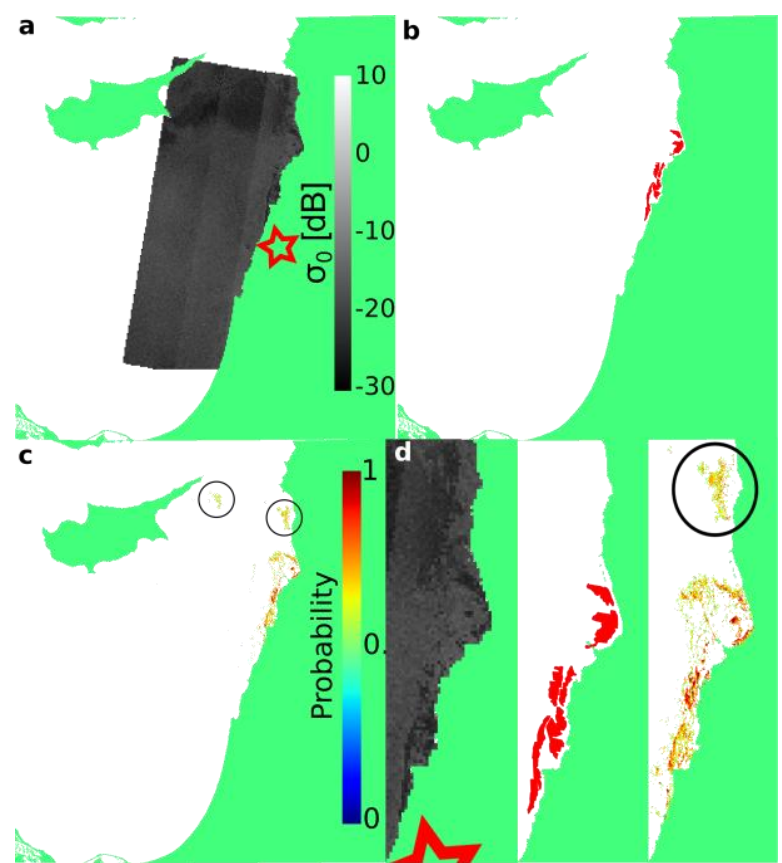

Figure 6. a) August 3 amplitude image. b) ESA Envisat ASAR and Modis August 3-4 spill map. c) Result with proposed algorithm. d) Zoom-in of the oil slick. Circles indicate differences.

As seen from Figures 5 and 6, the algorithm captures possible oil slicks rather well. In Figure 5, the results for each individual day are shown. In Figure 6, the amplitude for the August 3 image is shown with independent analysis of the same data, as well as the results obtained by the current algorithm (ZKI-DLR, 2006). There are possible artefacts in the northern part of the imaged area (highlighted with circles), which would likely been resolved if the stack had an earlier image. Nonetheless the areas with high oil slick probability in $\mathrm{b}$ and $\mathrm{c}$ match quite well, as shown in $\mathrm{d}$.

These results are compared to results of a machine learning technique based on a supervised classifier (Ozkan et al., 2013). The approach consists of the supervised artificial neural network binary classification of the textural features from the same SAR data of the same area with this study. However, an extrinsic comparison may be done because of the different algorithmic structures of these two approaches.

The advantages of the fully automatic algorithm are that there is no need for training areas and to adjust classifier parameters and it has ability of using different SAR data easily. Its main disadvantage is the more SAR data requirement to exclude the adverse atmospheric effects. Thus, more imagery data more accurate joint probabilities. The semi automatic approach is more user dependent and more data dependent. Preparation of training areas and classifier is more time consuming and more burdening. Although the performance of the semi automatic approach is slightly better for northern regions (Figure 7), the fully automatic approach may influence more strongly the scientific literature. 


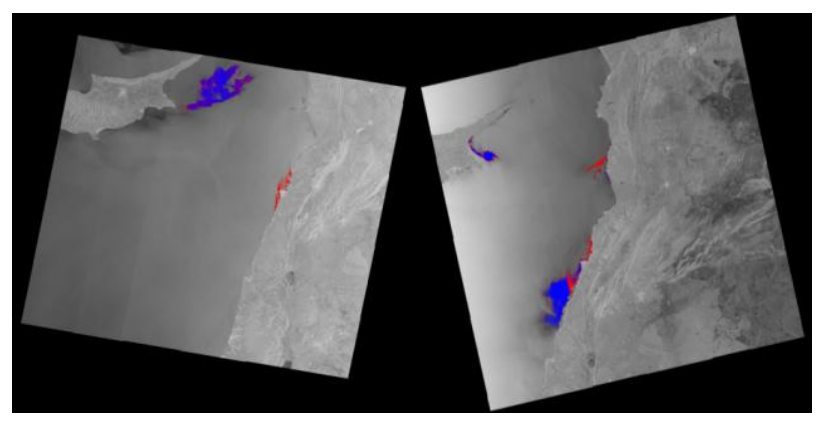

Figure 7. Result with semi-automatic approach (Red: oil, Blue: look-alike) (left) 2006/08/03, (right) 2006/08/05.

\section{CONCLUSION}

In this study, a fully automatic oil spill detection method is developed. It is based on changes in the radar cross section of surfaces under different conditions. The method further utilizes the temporal behavior of an oil spill to reduce the false positives due to natural variations in the radar backscatter, such as low wind speeds and naturally occurring slicks.

It is clear that the combination of multiple data sources can help minimize uncertainties. The proposed method not only presents a working example, but also an algorithm that can be easily expanded to introduce probabilities obtained from any data source available. This is a key property that increases the operational value of our automated oil spill detection algorithm.

\section{ACKNOWLEDGEMENTS}

The authors would like to thanks ESA for the Envisat ASAR data through CAT-1 \#11482.

\section{REFERENCES}

Alpers, W. and Huhnerfuss, H., 1988. Radar signatures of oil films floating on the sea surface and the marangoni effect. Journal of Geophysical Research 93(C4), pp. 3642-3648.

Assilzadeh, H and Mansor, S B 2001 Early warning system for oil spill using SAR images. Proceedings of ACRS 2001-22nd Asian Conference on Remote Sensing, Singapore, November 59, p 460-465

Barni, M., Betti, M. and Mecocci, A., 1995. A fuzzy approach to oil spill detection an sar images. In: Geoscience and Re- mote Sensing Symposium, 1995. IGARSS'95.'Quantitative Re- mote Sensing for Science and Applications', International, Vol. 1, IEEE, pp. 157-159.

Bragg, W., 1913. The diffraction of short electromagnetic waves by a crystal. In: Proceedings of the Cambridge Philosophical Society, Vol. 17, pp. 43-57.

Brekke, C. and Solberg, A., 2005. Oil spill detection by satellite remote sensing. Remote Sensing of Environment 95(1), pp. 113.

Cheng, Y., Li, X., Xu, Q., Garcia-Pineda, O., Andersen, O. and Pichel, W., 2011. Sar observation and model tracking of an oil spill event in coastal waters. Marine pollution bulletin 62(2), pp. 350-363.
Del Frate, F., Petrocchi, A., Lichtenegger, J. and Calabresi, G., 2000. Neural networks for oil spill detection using ers-sar data. Geoscience and Remote Sensing, IEEE Transactions on 38(5), pp. 2282-2287.

Espedal, H., 1999. Satellite sar oil spill detection using wind his- tory information. International Journal of Remote Sensing 20(1), pp. 49-65.

Fiscella, B., Giancaspro, A., Nirchio, F., Pavese, P. and Trivero, P., 2000. Oil spill detection using marine sar images. International Journal of Remote Sensing 21(18), pp. 3561-3566.

Gade, M., Alpers, W., Huhnerfuss, H., Wismann, V. and Lange, P., 1998. On the reduction of the radar backscatter by oceanic surface films: Scatterometer measurements and their theoretical interpretation. Remote Sensing of Environment 66(1), pp. 5270.

Haralick, R M, Shanmugam, K and Dinstein, I 1973 Textural features for image classification. IEEE Transactions on Systems, Man, and Cybernetics, 3(6) 610-621

Kim, D., Moon, W. and Kim, Y., 2010. Application of terrasar$\mathrm{x}$ data for emergent oil-spill monitoring. Geoscience and Remote Sensing, IEEE Transactions on 48(2), pp. 852-863.

Kubat, M., Holte, R. and Matwin, S., 1998. Machine learning for the detection of oil spills in satellite radar images. Machine learning 30(2), pp. 195-215.

Lombardini, P., Fiscella, B., Trivero, P., Cappa, C. and Garrett, W., 1989. Modulation of the spectra of short gravity waves by sea surface films: slick detection and characterization with a microwave probe. Journal of Atmospheric and Oceanic Technology 6, pp. 882-890.

Migliaccio, M., Nunziata, F. and Gambardella, A., 2009. On the co-polarized phase difference for oil spill observation. International Journal of Remote Sensing 30(6), pp. 1587-1602.

Ozkan, C. and Sunar, F., 2007. Comparisons of different semiautomated techniques for oil spill detection: A case study in lebanon. In: Mario A. Gomarasca (ed.), 27th EARSeL Symposium on GeoInformation, Millpress Rotterdam.

Ozkan, C., Osmanoglu, B. and Sunar, F., 2013.Efficiency of Envisat ASAR datasets in semi-automatic slick detection algorithm for Jiyeh Power Station oil spill, Lebanon. 35th International Symposium on Remote Sensing of Environment, Beijing China.

Solberg, A., Brekke, C. and Husoy, P., 2007. Oil spill detection in radarsat and envisat sar images. Geoscience and Remote Sensing, IEEE Transactions on 45(3), pp. 746-755.

Wessel, P. and Smith, W., 1996. A global, self-consistent, hierar- chical, high-resolution shoreline database. Journal of Geophysical Research 101, pp. 8741-8743.

ZKI-DLR, 2006. "Oil spill extent on August 3 and 4, 2006". Online: http://www.zki.dlr.de/map/507 